\title{
Research Paper: The Positive Effect of MiR1 Antagomir on Ischemic Neurological Disorders Via Changing the Expression of Bcl-w and Bad Genes
}

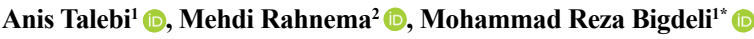 \\ 1. Department of Biology, Faculty of Life Sciences and Biotechnology, Shahid Beheshti University, Tehran, Iran. \\ 2. Department of Biology, Faculty of Engineering and Basic Sciences, Zanjan Branch, Islamic Azad University, Zanjan, Iran.
}

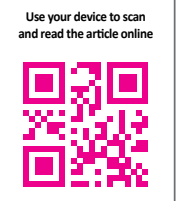

Citation: Talebi, A., Rahnema, M., \& Bigdeli, M. R. (2020). The Positive Effect of MiR1 Antagomir on Ischemic Neurological Disorders Via Changing the Expression of Bcl-w and Bad Genes. Basic and Clinical Neuroscience, 11(6), 811-820. http://dx.doi. org/10.32598/ben.11.6.324.3

http://dx.doi.org/10.32598/ben.11.6.324.3

\section{Article info:}

Received: 26 Feb 2018

First Revision: 10 Mar 2018

Accepted: 15 Oct 2019

Available Online: 01 Nov 2020

Keywords:

MiR1, Antagomir, Stroke, Bcl-w, Bad, Apoptosis, Infarct volume

\begin{abstract}
$\underline{\text { A B S T RA C T }}$
Introduction: MicroRNAs (miRNAs or miRs) are non-coding RNAs. Studies have shown that miRNAs are expressed aberrantly in stroke. The miR1 enhances ischemic damage, and a previous study has demonstrated that reduction of miR1 level has a neuroprotective effect on the Middle Cerebral Artery Occlusion (MCAO). Since apoptosis is one of the important processes in neural protection, the possible effect of miR1 on this pathway has been tested in this study. Post-ischemic administration of miR1 antagomir reduces infarct volume via bcl-w and bad expression.
\end{abstract}

Methods: Rats were divided into four experimental groups: sham, control, positive control, and antagomir treatment group. One hour after MCAO surgery, the rats were received intravenously (Tail vein) $0.1 \mathrm{~mL}$ Normal Saline (NS), $0.1 \mathrm{~mL}$ rapamycin, and $300 \mathrm{pmol} / \mathrm{g}$ miR1 antagomir (soluble in $0.1 \mathrm{~mL}$ normal saline) in control, positive control, and treatment group, respectively. Twenty-four hours after reperfusion infarct volume was measured. The expression of miR1, bcl-w, and bad were analyzed using real-time PCR in sham, control, and treated groups.

Results: Our results indicate that administration of miR1 antagomir reduces infarct volume significantly, it also decreases miR1 and bad expression while increases bcl-w expression.

Conclusion: Understanding the precise neuroprotective mechanism of miR1 antagomir can make it a proper treatment and an innovative approach for stroke therapy.

\section{* Corresponding Author:}




\section{Highlights}

- Our results indicate that administration of miR1 antagomir reduces MiR1 signicantly.

- The administration of miR1 antagomir reduces infarct volume and stroke signicantly.

- The administration of miR1 antagomir also decreases bad expression while increases BCL-W expression.

\section{Plain Language Summary}

Sroke is the main cause of death and disability and different genes, RNAs, and proteins are involved in the development of stroke. MicroRNAs are a type of small and non-coding RNAs. Many miRs exist in families. The miR1 microRNA precursor is a small miRNA that regulates its target protein's expression in the cell. In mammals, specific miRNAs have been recognized to control processes of differentiation, hematopoiesis, exocytosis, development, neuronal cell fate, apoptosis, and proliferation. MiR expression has been detected in stroke, Alzheimer disease, Parkinson disease, Down syndrome, and schizophrenia. It has been revealed that miR1 has a biological and pathological role in apoptosis, myocardial ischemic injures, contraction, myogenesis, and hypertrophy; also miR1related to skeletal and heart muscles in particular. Recently, it has been demonstrated that miR1 controls brain growth

\section{Introduction}

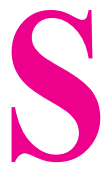

troke is one of the leading causes of death and long-term disability worldwide (Xua, Ouyanga, Xiong, Stary, \& Giffard, 2015). Numerous studies have demonstrated that different genes, RNAs, and proteins are involved in the development of stroke (Wang, Wang, \& Yang, 2013). Although many clinical trials have been tried, the only clinically efficacious treatment to date is thrombolysis (Blakeley \& Llinas, 2007). The complex interplay between multiple signaling pathways and intracellular organelles, the interaction between different cell types, and the potentially short therapeutic window for neuroprotection after stroke are suggested reasons for failures (Xua et al., 2015).

MicroRNAs (miRs or miRNAs) are a type of small, and non-coding RNAs. Mature miRs are generated from primary miR transcripts by sequential endonucleolytic processing and act as posttranscriptional regulators of gene expression, including in the setting of cerebral ischemia (Ouyang, Stary, Yang, \& Giffard, 2013).

Many miRs exist in families (Bigdeli, Rahnema, \& Khoshbaten, 2009). The miR1 microRNA precursor is a small miRNA that regulates its target protein's expression in the cell. MicroRNAs are transcribed as $\sim 70$ nucleotide precursors and subsequently processed by the Dicer enzyme to give $\sim 22$ nucleotide products. In this case, the mature sequence comes from the 3' arm of the precursor. The mature products are thought to have regulatory roles through complementarity to mRNA. Rno-miR-1 (special rat) has two mature products of rno-miR-1-3p and rnomiR1-5p. In this research, rno-miR-1-3p was considered.

It has been predicted that $40 \%$ to $50 \%$ of mammalian mRNAs could be regulated at the translational level by miRNAs (Jeyaseelan, Lim, \& Armugam, 2008). In mammals, specific miRNAs have been recognized to control processes of differentiation, hematopoiesis, exocytosis, development, neuronal cell fate, apoptosis, proliferation as well as in diseases (Kloosterman \& Plasterk, 2006) and possibly neuronal disorders (Kosik, 2006). MiR expression has been detected in stroke (Jeyaseelan et al., 2008; Dharap, Bowen, Place, \& Vemuganti, 2009), Alzheimer disease (Hebert et al., 2008), Parkinson disease (Kim et al., 2007), Down syndrome (Kuhn et al., 2008), and schizophrenia (Beveridge et al., 2008). Recent studies have identified stroke-induced miRNA in the brain and plasma from experimental models and patients (Dharap et al., 2009; Jeyaseelan et al., 2008).

It has been revealed that miR1 has a biological and pathological role in apoptosis, myocardial ischemic injures, contraction, myogenesis, and hypertrophy, on skeletal and heart muscles in particular (Xu et al., 2007). Recently, it has been demonstrated that miR 1 controls brain growth, generation of synapses, brain learning, and memory by the expression of brain-derived neurotrophic factor (Varendi, Kumar, Härma, \& Andressoo, 2014). An increase of miR1 in CSF-derived profile of microRNAs in animal models of stroke and Parkinson disease has already been confirmed (Wang, Ji, Cheng, Chen, \& Bai, 2014). 


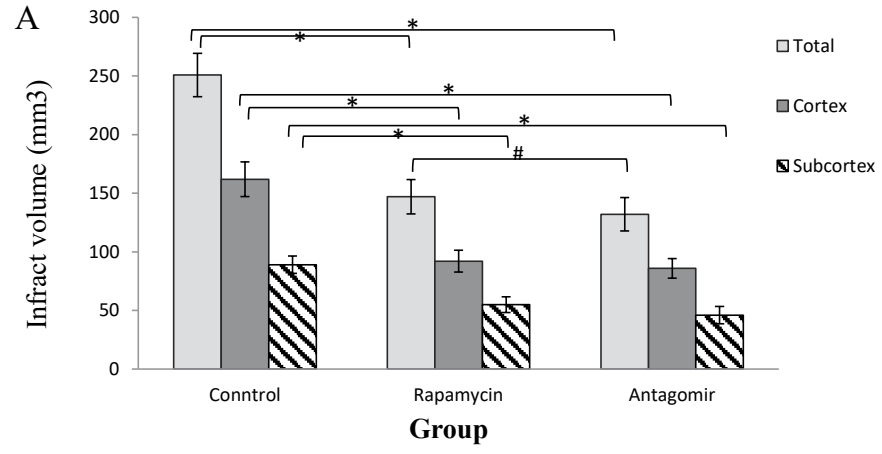

Figure 1. Effects of miR1 antagomir on infarct volume
B
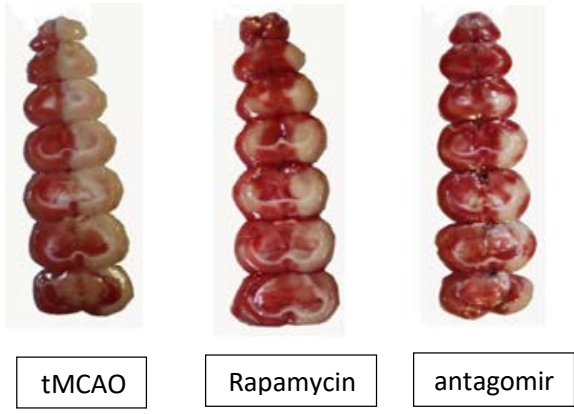

NEUR:SCIENCE

A: Effects of rapamycin, miR1 antagomir, and temporary middle cerebral artery occlusion (tMCAO) on infarct volume; B: Typical ischemic lesion induced by MCAO in rapamycin, miR1 antagomir and tMCAO groups; * $\mathrm{P}<0.05$; \# $\mathrm{P}<0.001$.

The mature miRNA such as miR1 binds to specific regions of target mRNA transcripts and either destabilize the target mRNA transcript or blocks its translation or both (Negrini, Nicoloso, \& Calin, 2009; Ghilidiyal \& Zamore, 2009; Brodersen \& Voinnet, 2009). In 2012, Selvamani et al. reported that cortical infarct volume was significantly reduced by ICV injection of anti-miR1, as compared to the control group that received the scrambled oligonucleotides. It has been shown that anti-miR1 treatment, as late as four hours following ischemia, significantly reduces cortical infarct volume in adult female rats (Selvamani, Sathyan, Miranda, \& Sohrabji, 2012). Chia$\mathrm{Yu} \mathrm{Ch}$ et al. reported that miR1 can regulate gene expression which consequently mediates hypoxia-induced apoptotic insults to neurons in vitro (Chang et al., 2016).

Recent experiments have demonstrated that microRNAs regulate multiple BCL-2 family members, including both pro-apoptotic and anti-apoptotic proteins, and influences the outcome of cerebral ischemia (Ouyang, Stary, White, \& Giffard 2015; Blakeley \& Llinas, 2007). Bcl-w and bad are anti-apoptotic and pro-apoptotic genes, respectively, which are regulated with rno-miR$1-3 p$. Members of the bcl 2 family can act either as antiapoptotic or pro-apoptotic molecules based on the presence of their conserved regions which are termed bcl2 homology (BH) domains (Van Delft \& Huang, 2006).

Anti-apoptotic subtypes share up to the four regions of sequence homology (BH1-4) such as Bcl-2, Bcl-xL, and Bcl-w. The pro-apoptotic members of the Bcl-2 family can be divided into two functionally and structurally distinct categories. The BH3-only proteins (e.g. Bim, Puma, Bid, and Bad) have only the $\mathrm{BH} 3$ region of homology which are activated in response to various cellular stress (Puthalakath \& Strasser, 2002), in contrast,
Bax and Bak contain multiple $\mathrm{BH}$ domains $(\mathrm{BH} 1, \mathrm{BH} 2$, and $\mathrm{BH} 3$ ) and require downstream of $\mathrm{BH} 3$-only proteins to launch apoptosis (Cheng et al., 2001; Rathmell, Lindsten, Zong, Cinalli, \& Thompson, 2002).

The pro-survival activity of BCL-2 has been illustrated in both overexpression and gene targeting studies. Overexpression of pro-survival members induced by internal or external stimuli, protect cells against apoptosis (Cory, Huang, \& Adams, 2003), which show the important overlapping roles for these proteins. The BH3-only pro-apoptotic proteins can bind with high affinity to special anti-apoptotic molecules and induce apoptosis when overexpressed (Huang \& Strasser 2000). Bcl-w is highly expressed in the brain, colon, and testes (O'Reilly et al., 2001). The level of bcl-w has been shown to increase during neuronal development that shows it may play a crucial role in neural survival (Hamnér, Skoglösa, \& Lindholm, 1999). Bcl-w protects neurons against $\mathrm{Ca}^{2+}$ mediated injuries by inhibiting the release of cytochrome-c following MCAO (Yan, Chen, Chen, Minami, \& Pei, 2000).

In vitro study of cerebellar granule neurons has revealed that overexpression of the bad gene induces cell death, which can be partly blocked by insulin growth factor (Datta et al., 1997). Also, it has been shown that bad protein level increases in nerve growth factor (NGF) deprived sympathetic neurons (Aloyz et al., 1998).

A few miRNAs target multiple members of the BCL-2 family. MiR-497 also targets BCL-W in addition to BCL-2 in neuro-2A cells. Knockdown of miR-497, which targets both BCL-2 and BCL-W (Yin et al., 2010), has a protective effect on MCAO-induced neural death. The bcl-w protein is also regulated by miR-29-a (Ouyang \& Giffard, 2014). Bad, a downstream target of Akt, is greatly activated in miR-494 
hearts, as evidenced by an 8 to 10 fold increase in levels of phosphorylated Bad (Wang et al., 2010). Although many in vitro research studies focus on diverse roles of miR1 in neurons, its in vivo role in the brain and different diseases particularly stroke is still unknown. The exact mechanisms of miR1 which result in the improvement of stroke deficits are still unclear. While many studies have shown the role of different microRNAs on anti-apoptotic and pro-apoptotic proteins ( $\mathrm{Su}, \mathrm{Yang}, \mathrm{Xu}, \mathrm{Chen}, \& \mathrm{Yu}$, 2015), the effect of microRNAs on bad and bcl-w expression, especially in brain ischemia, is not clarified.

In the current study, we investigated the possible effect of miR1 on bad and bcl-w gene expression in the rat model of brain ischemia. Our data suggest that miR1 ameliorates the outcome of ischemia by changing the expression of genes that are associated with the apoptosis pathway.

\section{Methods}

\subsection{Animals}

All experimental procedures were done with the approval of the Ethics Committee of Shahid Beheshti University of Iran.

All male rats (weighing 250-350 g) were purchased from Pasteur Institute and were provided with standard food and water. All animals were kept in a similar condition of 12:12 hours of a dark-light cycle at $25^{\circ} \mathrm{C}$.

\subsection{Experimental protocol}

Rats were divided into three experimental groups: control, positive control, and treatment. First, Middle Cerebral Artery Occlusion (MCAO) surgery was performed in all groups and one hour later, the rats received $0.1 \mathrm{~mL}$ normal saline (NS), $0.1 \mathrm{~mL}$ rapamycin and $300 \mathrm{pmol} / \mathrm{g}$ (soluble in $0.1 \mathrm{~mL}$ normal saline) miR1 (Guangzhou RiboBio Co. turkey, > rno-miR-1-3p MIMAT0003125 5'-UGGAAUGUAAAGAAGUGUGUAU -3') antagomir, in tail vein, respectively. About 24 hours after reperfusion, the infarct volume was assessed (Bigdeli et al., 2008).

\subsection{Focal cerebral ischemia}

MCAO surgery was done as described by longa et. Briefly rats were anesthetized with chloral hydrate (Merck, Germany) (28.5 mg/kg) and 3-0 silicone-coated nylon suture was introduced through the external carotid artery under a microscopic surgery, and advanced into the internal carotid artery 20 to $22 \mathrm{~mm}$ beyond the carotid bifurcation. Mild resistance represented that the tip was lodged in the anterior cerebral artery so the blood flow to the MCA was blocked. After 60 minutes of ischemia, the suture was extracted and reperfusion was started. During surgery, the temperature was monitored and maintained at $37.0^{\circ} \mathrm{C}$ (Citizen-513w, CITIZEN) by surface heating and cooling.

\subsection{Infarct volume assessment}

Rats were decapitated and their brains were removed rapidly and kept in saline at $4^{\circ} \mathrm{C}$ for 15 minutes. Brains were cut from the frontal to temporal side with a thickness of $2 \mathrm{~mm}$. They were incubated in $2 \%$ of 2, 3, 5-triphenyl tetrazolium chloride solution at $37^{\circ} \mathrm{C}$ in a water bath for 15 minutes. Then, the slices were photographed with a digital camera (Nokia 6630, Finland).

Unstained areas were defined as damaged areas. The infarct volume of unstrained areas was measured by an image analysis software (Image Tools, National Institutes of Health). Infarct volume was calculated according to the previously described method of Swanson et al. (Bigdeli et al., 2008) corrected infarct volume $=$ left hemisphere volume - (right hemisphere volume - infarct volume).

\subsection{Positive control}

As it has been proven, rapamycin ameliorates deficits of brain ischemia and has neuroprotective effects, it is used as a positive control (Wang et al., 2010; Su et al al., 2015; Sørensen, Nygaard, Nielsen, Jensen, \& Christensen, 2014).

\subsection{Primers design}

Primers were designed in the form of exon junction using allele ID software according to the sequence of genes archives in NCBI.

\section{7. rno-miR-1-3p gene}

\subsubsection{Looped primer sequence}

RT PRIMER: 5-GTCGTATCCAGTGCAGGGTCCGAGGTATTCGC

\section{ACTGGATACGACATACAC-3}

The primers forward: 5' $\rightarrow$ 3' GTGCAGGGTCCGAGGT

Reverse: 5' $\rightarrow$ 3' TGGAATGTAAAGAAGT 


\subsubsection{Bad gene}

The primers forward: 5' $\rightarrow$ 3' CGGAGTCGCCACAGTTCG

Reverse: 5' $\rightarrow$ 3'ACCCTCAAATTCATCGCTCATTC

\subsubsection{Bcl-w gene}

The primers forward: 5' $\rightarrow$ 3' ACTGGGGGCCCTGGTAACTGTAG

\section{Reverse: $5^{\prime} \rightarrow$ 3' CCACCCATCCACCAACTCCACT}

\subsubsection{Real-Time PCR}

The relative amount of the expression of miR1 gene in groups in comparison with GAPDH internal control was carried out using Corbet device.

\subsection{Statistical analysis}

Infarct volume was compared using 1-way ANOVA test (Newman-Keuls). Data were expressed as Means \pm SEM. $\mathrm{P}<0.05$ was considered significant.

\section{Results}

\subsection{Effects of miR1 antagomir on infarct volume}

The putative beneficial effects of rapamycin and miR1 antagomir were confirmed by a reduction in infarct volume when compared with the control group (Figure 1). The neuroprotection exerted by rapamycin and miR1 antagomir was mainly seen in the penumbra (cortex) and subcortex.

\subsection{Real-time PCR}

To study the quantitative level of gene expression, first, the technical accuracy was evaluated using the melting

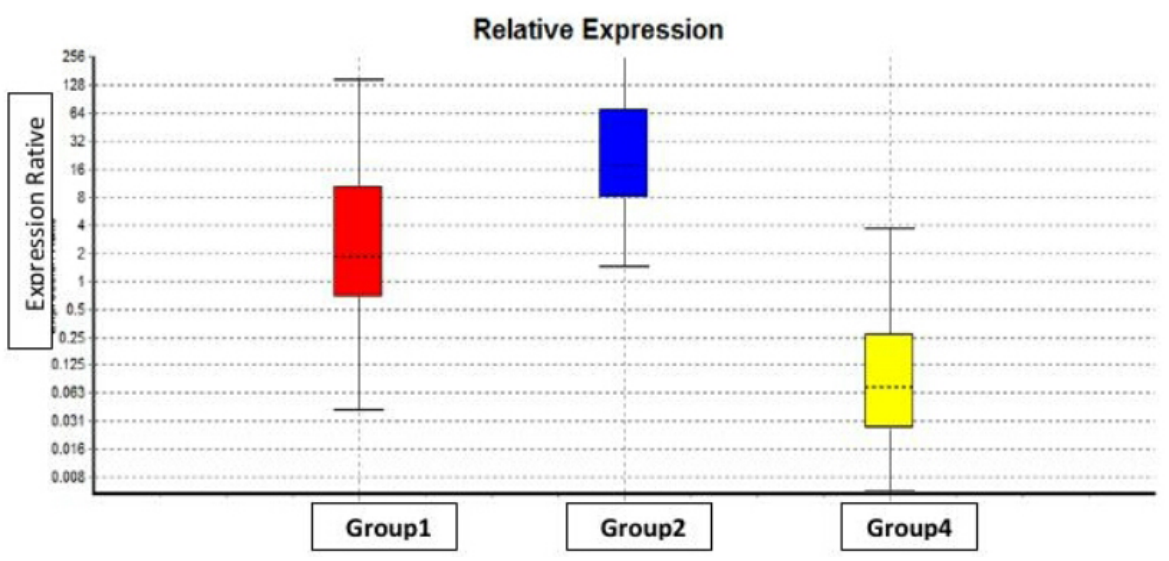

NEUR:SCIENCE

Figure 2. The ratio of miR1 gene expression in group 1 (sham) and group 2 (tMCAO) and group 4 (miR1 antagomir injection), 24 hours after injection

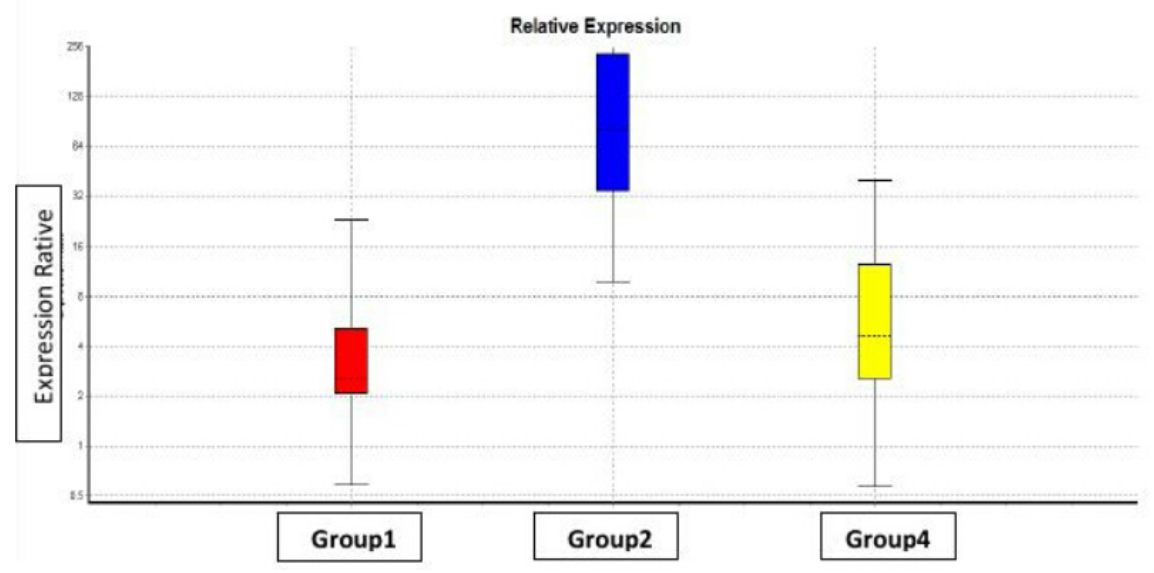

Figure 3. The ratio of Bad gene expression in group 1 (sham) and group 2 (tMCAO) and group 4 (miR1 antagomir injection), 24 hours after injection 


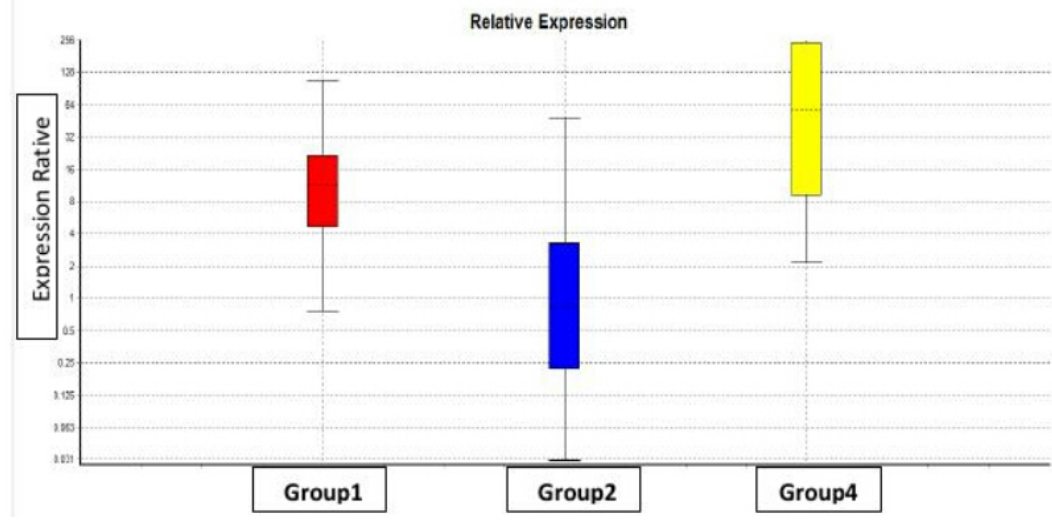

NEUR SCIENCE

Figure 4. The ratio of bcl-w gene expression in group 1 (sham) and group 2 (tMCAO) and group 4 (miR1 antagomir injection), 24 hours after injection

curve. Then the amount of CT was determined using the amplification diagram. Based on the results of the REST program, from the statistical point of view, the level of miR1, bad and bcl-w gene expression in the right hemisphere of experimental groups comes in Figures 2, 3, and 4 , respectively $(\mathrm{P} \leq 0.05)$.

\section{Discussion}

The main findings in this study are as follows: 1. Poststroke miR1 antagomir treatment has neuroprotective effects against ischemic neural damage compared to the control group in a rat model of MCAO; 2 . In vivo IV administration of miR1 has a neuroprotective effect; 3 . Administration of miR1 antagomir shortly after stroke results in a significant reduction in infarct volume; 4 . While miR1 expression increases after stroke, its expression decreases after intravenous injection of miR-1 antagomir; 5. Bad expression increases after stroke which has been reduced after intravenous injection of miR1 antagomir; and 6. bcl-w expression decreases after stroke which increased after intravenous injection of miR1 antagomir.

This study aims to investigate regulatory pathways that affect infarct volume in an animal model of MCAO, by focusing on gene expression which is regulated by miRNAs. Many studies have proved that miRNAs have an important role in cerebral ischemia and reperfusion (Sørensen et al., 2014). In 2010, Vemoganti and Pharap have shown that almost 9 miRNAs have been changed obviously during ischemia/reperfusion (IP) in the rat brain (Sørensen et al., 2014).

Dong et al. (2015) have revealed that miR1 can block liver cancer. It has been indicated that overexpression of miR1 induces apoptosis in the liver hepatocellular carcinoma (HepG2) via downregulation of apoptosis inhibitor 5 (API5) (Tang et al., 2009). Yehua et al. have shown that miR1 increased significantly and facilitates apopto- sis in response to $\mathrm{H}_{2} \mathrm{O}_{2}$ in rat cardiomyocytes. It has been revealed that administration of $\mathrm{miR} 1$ oligonucleotide inhibitors results in a resistance to $\mathrm{H}_{2} \mathrm{O}_{2}$ throughout downregulation of $\mathrm{Bcl} 2$ via miR1 which is connected to the 3UTR special location on this gene (Tang et al., 2009).

In 2014, a review was published about the unregulated production of miRNAs and their roles in neurodegenerative diseases. Overproduction of miR1 in stroke has been studied in reference 18. Anti-miR1 treatment, as late as 4 hours following ischemia, significantly reduced cortical infarct volume in adult female rats (Wang et al., 2013).

As described before, the role of miR-R has been studied in various diseases, but its effect on stroke has not been investigated precisely. Therefore, to figure out the role of miR1, we evaluate the possible effect of IV administration of miR1 antagomir in an animal model of brain ischemia.

We revealed that injection of miR1 antagomir after ischemia reduces infarct volume significantly. Many research studies show the effect of post-stroke treatment with other individual miRNAs, but, to our knowledge, we are the first who test post-stroke treatment or IV administration of miR1 antagomir. MiR1 has various mechanisms to protect neurons. It has been shown that apoptosis plays a key role in physiological neurogenesis in neural development (Okouchi, Ekshyyan, Maracine, \& Aw, 2007). On the other hand, neural weakness to apoptotic insults can lead to neurodegenerative disorders (Jana, Hogan, \& Pahan, 2009). A variety of intrinsic and extrinsic signals regulate cell apoptosis (Goyal, 2001; Lin et al., 2012). Chia et al. reported that miR1 induces apoptosis via collaboration with Bax, mitochondria, and caspase protease, in hypoxia-induced apoptotic in neuro-2a cell (Chang et al., 2016). 
Antagomir of miR1 and Let7f induce neuroprotection via binding to the 3 UTRs sites of multiple IGF signaling pathway components (Selvamani et al., 2012). Yehua et al. (2009) reported that the level of miR1 is inversely correlated with Bcl-2 protein expression in cardiomyocytes of the I/R rat model. In vitro studies have revealed that administration of miR1 inhibitor, oligonucleotides, and results in remarkable resistance to $\mathrm{H}_{2} \mathrm{O}_{2}$. The potential binding site of miR1 in the Bcl-2 gene have been identified. MiR1 reduces Bcl-2 expression in the level of mRNA and protein. These data have proven that miR1 plays an important role in the regulation of cardiomyocyte apoptosis, which is involved in the post-transcriptional expression of Bcl-2 (Tang et al., 2009).

MiRs bind to messenger RNAs (mRNAs), and according to their sequence, results in a decrease or increase of their target genes translation (Ouyang \& Giffard, 2014).

Bcl-w (antiapoptotic) and Bad (proapoptotic) are two important targets of miR1 that have a critical role in the regulation of apoptosis. MiR1 has a positive and negative effect on bad and bcl-w expression, respectively (www.mirbase.org).

An increase in BCL-w expression induces neural survival in NGF-dependent trigeminal neurons and BDNF-dependent neurons in response to neurotrophin (Middleton, Wyatt, Ninkina, \& Davies, 2001). It is notable that, BCL$\mathrm{w}$ expression has increased in mouse neurons up to 72 hours after MCAO (Yan et al., 2000). These results show that BCL-w plays a critical role in different neurological conditions to protect neurons which makes it an attractive target for the development of new therapeutic agents.

Several studies have demonstrated that BCL-w interacts with the pro-apoptotic protein BAD and blocks apoptosis in sympathetic neurons (Hamnér et al., 2001). An increase in bad expression causes cell death in cultured cerebellar granule neurons which can be partially stopped by co-expression of an active form of the protein kinase B/Akt (Datta et al., 1997). Two isoforms of Bad can induce cell death of sympathetic neurons and $\mathrm{Bcl}-\mathrm{w}$ has a protective role both in Bad induced and NGF-withdrawal-induced cell death of these neurons (Hamnér et al., 2001).

Bad and bcl-w are two important members of the BCL-2 family which are regulated by different types of microRNAs. Recent studies have shown that miR-125-b (Gong et al., 2013), miR-29-a (Datta et al., 1997), miR-29b (Shi et al., 2012), and miR-497 regulate bcl-w (Yadav et al., 2011). Studies have demonstrated that various neuropro- tective treatments reduce the impact of stroke via increasing the expression levels of either bcl-2 or bcl-xL (Dubal, Shughrue, Wilson, Merchenthaler, \& Wise, 1999). Overall, these studies have indicated the importance of the antiapoptotic Bcl-2 family in ischemic cerebral injury, and thus make these proteins as possible neuroprotective substance against stroke (Broughton, Reutens, \& Sobey, 2009).

Because micro-RNA has a well-known role in ameliorating stroke outcomes via apoptosis regulation and also its exact protection pathway is still unclear, this study can be a new step for detecting new treatment strategies by evaluation of the possible effect of miR1 on bad and bcl-w expression.

Our real-time PCR data analysis of miR1 level revealed that miR 1 expression decreased significantly in the miR 1 antagomir treated group compared to the control group 24 hours after reperfusion by decreasing apoptosis in the rat model of MCAO. On the other hand, injection of miR1 antagomir increased and decreased mRNA level of bcl-w and bad, respectively at the same time.

MiR1 antagomir reduces infarct volume via changes in the expression of miR1 target genes. Our study focused on the biological function and pathways of miR1 target genes to understand their apoptotic or anti-apoptotic effects. Further investigation is needed to clarify the precise relationship between bad and bcl-w, as pro-apoptotic and anti-apoptotic factors.

According to the previous studies, microRNAs (miRNAs or miRs) are small, endogenous, single-stranded, and noncoding RNAs that have 18-25 nucleotides (Tang, 2005; Giannakakis, Coukos, Hatzigeoriou, Sandaltzopoulos, \& Zhang, 2007). They regulate gene expression by hybridization to mRNA post-transcriptionally, which lead to suppress or degrade target mRNA (Giannakakis et al., 2007; Van Rooij \& Olson, 2007). Because apoptosis has an important role in the hypoxic condition, any gene which is regulated by miR1 affects apoptosis. Finding a new way to regulate the miR1 pathway can ameliorate neurodegenerative diseases such as ischemia via apoptosis regulation.

This study not only represented a new possible treatment for brain ischemia (to salvage the ischemic penumbra) in vivo, but it also could help us to understand more about the pathology of this disease. 


\section{Ethical Considerations}

\section{Compliance with ethical guidelines}

This study was approved with the Ethics Committee of the Faculty of Biological Sceince of Shahid Beheshti University.

Funding

The paper was extracted from $\mathrm{PhD}$. thesis of first author in the Department of Biology, Faculty of Life Sciences and Biotechnology, Shahid Beheshti University, Tehran.

\section{Authors' contributions}

All authors contributed equally in preparing all parts of the research.

\section{Conflict of interest}

The authors declared no conflict of interest.

\section{References}

Aloyz, R. S., Bamji, S. X., Pozniak, C. D., Toma, J. G., Atwal, J., \& Kaplan, D. R., et al. (1998). p53 is essential for developmental neuron death as regulated by the TrkA and p75 neurotrophin receptors. The Journal of Cell Biology, 143(6), 1691-703. [DOI:10.1083/jcb.143.6.1691] [PMID] [PMCID]

Beveridge, N. J., Tooney, P. A., Carroll, A. P., Gardiner, E., Bowden, N., \& Scott, R. J., et al. (2008). Dysregulation of miRNA 181b in the temporal cortex in schizophrenia. Human Molecular Genetics, 17(8), 1156-68. [DOI:10.1093/hmg/ddn005] [PMID]

Bigdeli, M. R., Hajizadeh, S., Froozandeh, M., Heidarianpour A., Rasoulian, B., \& Asgari, A. R., et al. (2008). Normobaric hyperoxia induces ischemic tolerance and upregulation of glutamate transporters in the rat brain and serum TNF-c level. Experimental Neurology, 212(2), 298-306. [DOI:10.1016/j. expneurol.2008.03.029] [PMID]

Bigdeli, M. R., Rahnema, M., \& Khoshbaten, A. (2009). Preconditioning with sublethal ischemia or intermittent normobaric hyperoxia up-regulates glutamate transporters and tumor necrosis factor-a converting enzyme in the rat brain Journal of Stroke and Cerebrovascular Diseases, 18(5), 336-42. [DOI:10.1016/j.jstrokecerebrovasdis.2008.12.005] [PMID]

Blakeley, J. O., \& Llinas, R. H. (2007). Thrombolytic therapy for acute ischemic stroke. Journal of the Neurological Sciences, 261(1-2), 55-62. [DOI:10.1016/j.jns.2007.04.031] [PMID]

Brodersen, P., \& Voinnet, O. (2009). Revisiting the principles of microRNA target recognition and mode of action. Nature Reviews Molecular Cell Biology, 10(2), 141-8. [DOI:10.1038/ nrm2619] [PMID]
Broughton, B. R. S., Reutens, D. C., \& Sobey, C. G. (2009). Apoptotic mechanisms after cerebral ischemia. Stroke, 40(5), e331-9. [DOI:10.1161/STROKEAHA.108.531632] [PMID]

Chang, C. Y., Lui, T. N., Lin, J. W., Lin, Y. L., Hsing, C. H., \& Wang J. J., et al. (2016). Roles of microRNA-1 in hypoxia-induced apoptotic insults to neuronal cells. Archives of Toxicolooy, 90(1), 191202. [DOI:10.1007/s00204-014-1364-x] [PMID]

Cheng, E. H., Wei, M. C., Weiler, S., Flavell, R. A., Mak, T. W., \& Lindsten, T., et al. (2001). Bcl2, BclxL sequester BH3 domainonly molecules preventing BAX- and BAK-mediated mitochondrial apoptosis. Molecular Cell, 8(3), 705-11. [DOI:10.1016/S10972765(01)00320-3] [PMID]

Cory, S., Huang, D. C. S., \& Adams, J. M. (2003). The Bcl2 family: Roles in cell survival and oncogenesis. Oncogene, 22(53), 8590607. [DOI:10.1038/sj.onc.1207102] [PMID]

Datta, S. R., Dudek, H., Tao, X., Masters, S., Fu, H., \& Gotoh, Y., et al. (1997). Akt phosphorylation of BAD couples survival signals to the cell-intrinsic death machinery. Cell, 91(2), 231-41. [DOI:10.1016/S0092-8674(00)80405-5] [PMID]

Dharap, A., Bowen, K., Place, R., Li, L. C., \& Vemuganti, R. (2009). Transient focal ischemia induces extensive temporal changes in rat cerebral MicroRNAome. Journal of Cerebral Blood Flow \& Metabolism, 29(4), 675-87. [DOI:10.1038/jcbfm.2008.157] [PMID] [PMCID]

Dubal, D. B., Shughrue, P. J., Wilson, M. E., Merchenthaler, I., \& Wise, P. M. (1999). Estradiol modulates Bcl-2 in cerebral ischemia: A potential role for estrogen receptors. The Journal of Neuroscience, 19(15), 6385-93. [DOI:10.1523/JNEUROSCI.19-15-06385.1999] [PMID] [PMCID]

Giannakakis, A., Coukos, G., Hatzigeoriou, A., Sandaltzopoulos, R., \& Zhang, L. (2007). miRNA genetic alternations in human cancers. Expert Opinion on Biological Therapy, 7(9), 1375-86. [DOI:10.1517/14712598.7.9.1375] [PMID]

Gong, J., Zhang, J. P., Li, B., Zeng, C., You, K., \& Chen, M. X., et al. (2013). MicroRNA-125b promotes apoptosis by regulating the expression of Mcl-1, Bcl-w and IL-6R. Oncogene, 32(25), 3071-9. [DOI:10.1038/onc.2012.318] [PMID]

Goyal, L. (2001). Cell death inhibition: Keeping caspases in check Cell, 104(6), 805-8. [DOI:10.1016/S0092-8674(01)00276-8] [PMID]

Hamnér, S., Arumäe, U., Li-Ying, Y., Sun, Y. F., Saarma, M., \& Lindholm, D. (2001). Functional characterization of two splice variants of rat bad and their interaction with Bcl-w in sympathetic neurons. Molecular and Cellular Neuroscience, 17(1), 97-106. [DOI:10.1006/mcne.2000.0905] [PMID]

Hamnér, S., Skoglösa, Y., \& Lindholm, D. (1999). Differential expression of Bcl-w and Bcl-x messenger RNA in the developing and adult rat nervous system. Neuroscience, 91(2), 673-84. [DOI:10.1016/S0306-4522(98)00642-3]

Hebert, S. S., Horr'e, K., Nicolaï, L., Papadopoulou, A. S., Mandemakers, W., \& Silahtaroglu, A. N., et al. (2008). Loss of microRNA cluster miR-29a/b-1 in sporadic Alzheimer's disease correlates with increased BACE1/beta-secretase expression Proceedings of the National Academy of Sciences of the United States of America, 105(17), 6415-20. [DOI:10.1073/pnas.0710263105] [PMID] [PMCID]

Huang, D. C. S., \& Strasser, A. (2000). BH3-only proteins - essentia initiators of apoptotic cell death. Cell, 103, 839-42. [DOI:10.1016/ S0092-8674(00)00187-2 
Jana, A., Hogan, E. L., \& Pahan, K. (2009). Ceramide and neurodegeneration: Susceptibility of neurons and oligodendrocytes to cell damage and death. Journal of the Neurological Sciences, 278(12), 5-15. [DOI:10.1016/j.jns.2008.12.010] [PMID] [PMCID]

Jeyaseelan, K., Lim, K. Y., \& Armugam, A. (2008). MicroRNA expression in the blood and brain of rats subjected to transient focal ischemia by middle cerebral artery occlusion. Stroke, 39(3), 959-66. [DOI:10.1161/STROKEAHA.107.500736] [PMID]

Kim, J., Inoue, K., Ishii, J., Vanti, W. B., Voronov, S. V., \& Murchison, E., et al. (2007). A microRNA feedback circuit in midbrain dopamine neurons. Science, 317(5842), 1220-4. [DOI:10.1126/science.1140481] [PMID] [PMCID]

Kloosterman, W. P., \& Plasterk, R. H. A. (2006). The diverse functions of microRNAs in animal development and disease. Developmental Cell, 11(4), 441-50. [DOI:10.1016/j.devcel.2006.09.009] [PMID]

Kosik, K. S. (2006). The neuronal microRNA system. Nature Reviews Neuroscience, 7(12), 911-20. [DOI:10.1038/nrn2037] [PMID]

Kuhn, D. E., Nuovo, G. J., Martin, M. M., Malana, G. E., Pleister, A. P., \& Jiang, J., et al. (2008). Retracted: Human chromosome 21-derived miRNAs are overexpressed in Down syndrome brains and hearts. Biochemical and Biophysical Research Coтmunications, 370(3), 473-7. [DOI:10.1016/j.bbrc.2008.03.120] [PMID] [PMCID]

Li, D., Liu, Y., Li, H., Peng, J. J., Tan, Y., \& Zou, Q., et al. (2015) MicroRNA-1 promotes apoptosis of hepatocarcinoma cells by targeting Apoptosis Inhibitor-5 (API-5). FEBS Letters, 589(1), 6876. [DOI:10.1016/j.febslet.2014.11.025] [PMID]

Lin, J. W., Chen, J. T., Hong, C. Y., Lin, Y. L., Wang, K. T., \& Yao, C. J., et al. (2012). Honokiol traverses the blood-brain barrier and induces apoptosis of neuroblastoma cells via an intrinsic Baxmitochondrion-cytochrome c-caspase protease pathway. NeuroOncology, 14(3), 302-14. [DOI:10.1093/neuonc/nor217] [PMID] [PMCID]

Middleton, G., Wyatt, S., Ninkina, N., \& Davies, A. M. (2001). Reciprocal developmental changes in the roles of Bcl-w and Bcl$x(\mathrm{~L})$ in regulating sensory neuron survival. Development, 128(3), 447-57. [PMID]

Negrini, M., Nicoloso, M. S., \& Calin, G. A. (2009). MicroRNAs and cancer-new paradigms in molecular oncology. Current Opinion in Cell Biology, 21(3), 470-9. [DOI:10.1016/j.ceb.2009.03.002] [PMID]

Okouchi, M., Ekshyyan, O., Maracine, M., \& Aw, T. Y. (2007). Neuronal apoptosis in neurodegeneration. Antioxidants $\mathcal{E}$ Redox Signaling, 9(8), 1059-96. [DOI:10.1089/ars.2007.1511] [PMID]

O'Reilly, L. A., Print. C., Hausmann, G., Moriishi, K., Cory. S., \& Huang, D. C., et al. (2001). Tissue expression and subcellular localization of the pro-survival molecule Bcl-w. Cell Death \& Differentiation, 8(5), 486-94. [DOI:10.1038/s..cdd.4400835] [PMID]

Ouyang, Y. B., \& Giffard, R. G. (2014). MicroRNAs affect BCL-2 family proteins in the setting of cerebral ischemia. Neurochemistry International, 77, 2-8. [DOI:10.1016/j.neuint.2013.12.006] [PMID] [PMCID]

Ouyang, Y. B., Stary, C. M., White, R. E., \& Giffard, R. G. (2015). The use of microRNAs to modulate redox and immune response to stroke. Antioxidants \& Redox Signaling, 22(2), 187202. [DOI:10.1089/ars.2013.5757] [PMID] [PMCID]
Ouyang, Y. B., Stary, C. M., Yang, G. Y., \& Giffard, R. (2013). microRNAs: Innovative targets for cerebral ischemia and stroke. Current Drug Targets, 14(1), 90-101. [DOI:10.2174/1 38945013804806424] [PMID] [PMCID]

Puthalakath, H., \& Strasser, A. (2002). Keeping killers on a tight leash: Transcriptional and post-translational control of the pro-apoptotic activity of BH3-only proteins. Cell Death $\mathcal{E}$ Differentiation, 9(5), 505-12. [DOI:10.1038/sj.cdd.4400998] [PMID]

Rathmell, J. C., Lindsten, T., Zong, W. X., Cinalli, R. M., \& Thompson, C. B. (2002). Deficiency in BAK and BAX perturbs thymic selection and lymphoid homeostasis. Nature Immunology, 3(10), 932-9. [DOI:10.1038/ni834] [PMID]

Shi, G., Liu, Y., Liu, T., Yan, W., Liu, X., \& Wang, Y., et al. (2012). Upregulated miR-29b promotes neuronal cell death by inhibiting Bcl2L2 after ischemic brain injury. Experimental Brain Research, 216(2), 225-30. [DOI:10.1007/s00221-0112925-3] [PMID]

Sørensen, S. S., Nygaard, A. B., Nielsen, M. Y., Jensen, K., \& Christensen, T. (2014). miRNA expression profiles in cerebrospinal fluid and blood of patients with acute ischemic stroke. Translational Stroke Research, 5(6), 711-8. [DOI:10.1007/s12975-014-0364-8] [PMID]

Su, Z., Yang, Z., Xu, Y., Chen, Y., \& Yu, Q. (2015). MicroRNAs in apoptosis, autophagy and necroptosis. Oncotarget, 6(11) 8474-90. [DOI:10.18632/oncotarget.3523] [PMID] [PMCID]

Tang, G. (2005). siRNA and miRNA: An insight into RISCs. Trends in Biochemical Sciences, 30(2), 106-14. [DOI:10.1016/j. tibs.2004.12.007] [PMID]

Tang, Y., Zheng, J., Sun, Y., Wu, Z., Liu, Z., \& Huang, G. (2009). MicroRNA-1 regulates cardiomyocyte apoptosis by targeting Bcl-2. International Heart Journal, 50(3), 377-87. [DOI:10.1536/ihj.50.377] [PMID]

van Delft, M. F., \& Huang, D. C. S. (2006). How the Bcl2 family of proteins interact to regulate apoptosis. Cell Research, 16(2) 203-13. [DOI:10.1038/sj.cr.7310028] [PMID]

van Rooij, E., \& Olson, E. N. (2007). MicroRNAs: Powerful new regulators of heart disease provocative therapeutic targets. The Journal of Clinical Investigation, 117(9), 2369-76. [DOI:10.1172/JCI33099] [PMID] [PMCID]

Varendi, K., Kumar, A., Härma, M. A., \& Andressoo, J. O. (2014). miR1, miR10b, miR155, and miR191 are novel regulators of BDNF. Cellular and Molecular Life Sciences, 71(22) 4443-56. [DOI:10.1007/s00018-014-1628-x] [PMID] [PMCID]

Wang, C., Ji, B., Cheng, B., Chen, J., \& Bai, B. (2014). Neuroprotection of microRNA in neurological disorders (review). Biomedical Reports, 2(5), 611-9. [DOI:10.3892/br.2014.297] [PMID] [PMCID]

Wang, X., Zhang, X., Ren, X. P., Chen, J., Liu, H., \& Yang, J., et al. (2010). MicroRNA-494 targeting both proapoptotic and antiapoptotic proteins protects against ischemia/reperfusion-induced cardiac injury. Circulation, 122(13), 1308-18 [DOI:10.1161/CIRCULATIONAHA.110.964684] [PMID] [PMCID]

Wang, Y., Wang, Y., \& Yang, G. Y. (2013). MicroRNAs in cerebral ischemia. Stroke Research and Treatment, 2013, 276540. [DOI:10.1155/2013/276540] [PMID] [PMCID] 
Xu, C., Lu, Y., Pan, Z., Chu, W., Luo, X., \& Lin, H., et al. (2007). The muscle-specific microRNAs miR-1 and miR-133 produce opposing effects on apoptosis by targeting HSP60, HSP70 and caspase-9 in cardiomyocytes. Journal of Cell Science, 120(Pt 17), 3045-52. [DOI:10.1242/jcs.010728] [PMID]

Xua, L. J., Ouyanga, Y. B., Xiong, X., Stary, C. M., \& Giffard, R. G. (2015). Post-stroke treatment with miR-181 antagomir reduces injury and improves long-term behavioral recovery in mice after focal cerebral ischemia. Experimental Neurology, 264, 1-7. [DOI:10.1016/j.expneurol.2014.11.007] [PMID] [PMCID]

Yadav, S., Pandey, A., Shukla, A., Talwelkar, S. S., Kumar, A., \& Pant, A. B., et al. (2011). miR-497 and miR-302b regulate ethanol-induced neuronal cell death through BCL2 protein and cyclin D2. Journal of Biological Chemistry, 286(43), 3734757. [DOI:10.1074/jbc.M111.235531] [PMID] [PMCID]

Yan, C., Chen, J., Chen, D., Minami, M., \& Pei, W. (2000). Overexpression of the cell death suppressor Bcl-w in ischemic brain Implications for a neuroprotective role via the mitochondrial pathway. Journal of Cerebral Blood Flow \& Metabolism, 20(3), 620-30. [DOI:10.1097/00004647-200003000-00020] [PMID]

Yin, K. J., Deng, Z., Huang, H., Hamblin, M., Xie, C., \& Zhang, J., et al. (2010). miR-497 regulates neuronal death in mouse brain after transient focal cerebral ischemia. Neurobiology of Disease, 38(1), 17-26. [DOI:10.1016/j.nbd.2009.12.021] [PMID] [PMCID] 